\title{
Extracting Quantitative Information on Coastal Ice Dynamics and Ice Hazard Events From Marine Radar Digital Imagery
}

\author{
Rohith MV, Member, IEEE, Joshua Jones, Hajo Eicken, and Chandra Kambhamettu
}

\begin{abstract}
Marine radars have been employed to gather data in applications that require near-continuous monitoring and tracking of objects over a wide area from a single viewpoint, independent of weather and light conditions. However, little attention has been paid toward utilizing such systems for the study of long-term phenomena and detecting anomalous environmental events or hazards that may occur infrequently but have potentially significant impacts on coastal populations. In this paper, we concentrate on tracking features in seasonally ice-covered Arctic coastal ocean environments. We have developed tools for automated analysis of ground-based radar images of landfast ice and moving sea ice to extract ice-floe trajectories and velocity fields, delineate the boundary of stable landfast ice, detect events relevant to coastal populations and identify surface vessels. We employ dense and feature-based optical flow approaches to compute motion fields from the images, active contours for delineation of stable landfast ice, and Hidden Markov Models for machine learning based event detection. We present results from the analysis of sample images jointly with a quantitative evaluation of algorithm performance relative to operator-based assessments.
\end{abstract}

Index Terms-Deformable contours, marine radar, motion tracking, optical flow, sea ice.

\section{INTRODUCTION}

$\mathbf{M}$ ARINE radars have been employed to gather data in applications that require near-continuous monitoring of a wide area from a single viewpoint, independent of weather and light conditions [1], [2]. Though tracking objects in radar imagery has been explored thoroughly (e.g., [1], [3]), much less attention has been paid toward utilizing such systems for the study of long-term phenomena and detecting anomalous environmental events or hazards that may occur infrequently but have potentially significant impacts on coastal populations.

Manuscript received February 1, 2011; revised October 7, 2011; accepted March 9, 2012. Date of publication November 22, 2012; date of current version April 18, 2013. This work was supported through the Department of Homeland Security's Centers of Excellence Program (Center for Island, Maritime, and Extreme Environment Security at University of Hawaii). Support by the Alaska Ocean Observing System and the National Science Foundation helped put key components of the ice observatory that this work builds on in place. Any opinions, findings, and conclusions or recommendations expressed in this material are those of the authors and do not necessarily reflect the views of the agencies that funded this work.

R. MV and C. Kambhamettu are with the Department of Computer and Information Sciences, University of Delaware, Newark, DE 19716 USA (e-mail: rohithmv@udel.edu; chandrak@udel.edu).

J. Jones and H. Eicken are with Geophysical Institute, University of Alaska Fairbanks, Fairbanks, AK 99775 USA.

Color versions of one or more of the figures in this paper are available online at http://ieeexplore.iee.org.

Digital Object Identifier 10.1109/TGRS.2012.2217972
Since the acquired data is sensitive to reflector characteristics (which can change rapidly) and occlusions, typical computer vision techniques alone do not suffice for analyzing such imagery. The phenomena observed and the products that are derived from the imagery are domain and task-specific and hence, so must be the techniques employed in radar image analysis.

In this paper, we concentrate on tracking features in seasonally ice-covered Arctic Ocean coastal environments. As a result of changes in the ice cover characteristics and increasing industrial activity, these regions have experienced substantial increases in maritime traffic [4], posing challenges to agencies and operators engaged in ensuring safety of maritime operations [5]. At the same time, traditional uses of ice environments by coastal communities can also benefit substantially from improved ice hazard tracking for personal safety [6]. Here, we describe an automated approach for analyzing imagery from a prototype coastal marine radar deployed at Barrow, Alaska to track the extent of stable landfast ice, detect ice break-out events and ice hazards, as well as identify surface vessels. We use a combination of existing and novel methods for analyzing radar imagery and develop a framework in which the characteristics corresponding to the different events may be identified and tracked for eventual ingestion into decision support tools. The goal of this work is to work toward a system that can provide supplemental information in assessing potentially hazardous situations or track surface vessels by human operators.

We employ the following: 1) dense and feature-based optical flow approaches to compute motion fields from the images; 2) active contours for delineation of stable landfast ice; and 3) a Hidden Markov Model (HMM) for machine-learning based identification of the key events. Analysis and manual validation of the results obtained indicate that the system performs as desired. In the next section, we provide background information on the need for an automated, ground-based radar system for the analysis of ice dynamics and hazards in coastal Arctic waters, and introduce the details of our radar setup. In Sections III and IV, we discuss feature selection and deformable model analysis as a background to our approach. In Section V, we outline the general approach, algorithms employed, and the image-derived products. Sections VI-IX describe the details of estimating motion fields, ice stability, feature detection and evaluation of anomalous events. In Section X, we present results from the analysis of sample images jointly with a quantitative evaluation of algorithm performance relative to operator-based assessments. The paper closes with conclusions and discussion of next steps (Section XI). 


\section{Ground-Based Radar Systems to Track Surface Processes and ANOMAlous Events-Motivation AND DETAILS OF BARROW ICE OBSERVATORY}

Ground-based radar systems offer several advantages in tracking surface processes or features compared to airborne or space based radars. These advantages include much higher sampling rates, stable configuration, and short path delays in a system that is much more cost-effective. However, groundbased radar is more prone to occlusion due to the lower elevation of the system, with a range typically constrained by emitted power and/or the radar horizon [1]. For applications such as those described here that are focused on a specific locale, this is an acceptable drawback. Moreover, networked radar systems or readily deployed field units can help provide better coverage over the area of interest. Ground-based radar has been employed successfully to observe a range of surface processes in land and ocean environments. Werner et al. [7] installed a Ku-band radar at the Rhône Glacier, Switzerland. It consists of a real aperture interferometer which can be used to generate an elevation map of the glacier with meter scale accuracy and also to monitor large scale changes due to glacier melt or dynamics. There have been similar attempts at in situ study of landslides using ground-based radar [8], [9]. In polar marine environments, ground-based radar systems are in wide use for maritime navigation, and in particular, the detection of ice-associated hazards [1], [10]. Coastal radar systems have also been employed in the context of ensuring safe navigation and tracking ice hazards, as well as long-term changes in the ice cover. One of the most sophisticated systems has been deployed off northern Japan, where ice was monitored along $250 \mathrm{~km}$ of coastline for a distance of $50 \mathrm{~km}$ out to sea for more than three decades [11].

At Barrow, Alaska, coastal radars have been employed to monitor and track the seasonal evolution of the ice cover [12]. However, most if not all of this work has relied on manual analysis of radar imagery by operators to track ice movement, identify features, or evaluate hazards. One of the very few studies attempting a quantitative approach that we are aware of is the work by Kimura et al. (unpublished), employing cross-correlation techniques to derive motion fields for the Hokkaido radar system. However, such standard image analysis approaches are not always effective in working with radar imagery, as they are hampered by the lack of persistent scatterers, variations in radar signal return strength, and lack of scatterers over smooth ice. Also, analysis of radar imagery for potential ocean or ice hazards and tracking of features such as vessels in the context of decision-support systems requires the extraction of information that goes beyond gridded velocity fields. These shortcomings provide the motivation for our present study, which aims to lay the groundwork for automated, operational processing, and analysis of imagery obtained from a radar system to: 1) aid in decision-making by operators and others concerned with safety of maritime and coastal operations; and 2) allow for automated extraction of sea-ice velocity fields that allow for derivation of ice dynamics parameters complementary to information obtained from analysis of satellite data (e.g., [13], [14]).

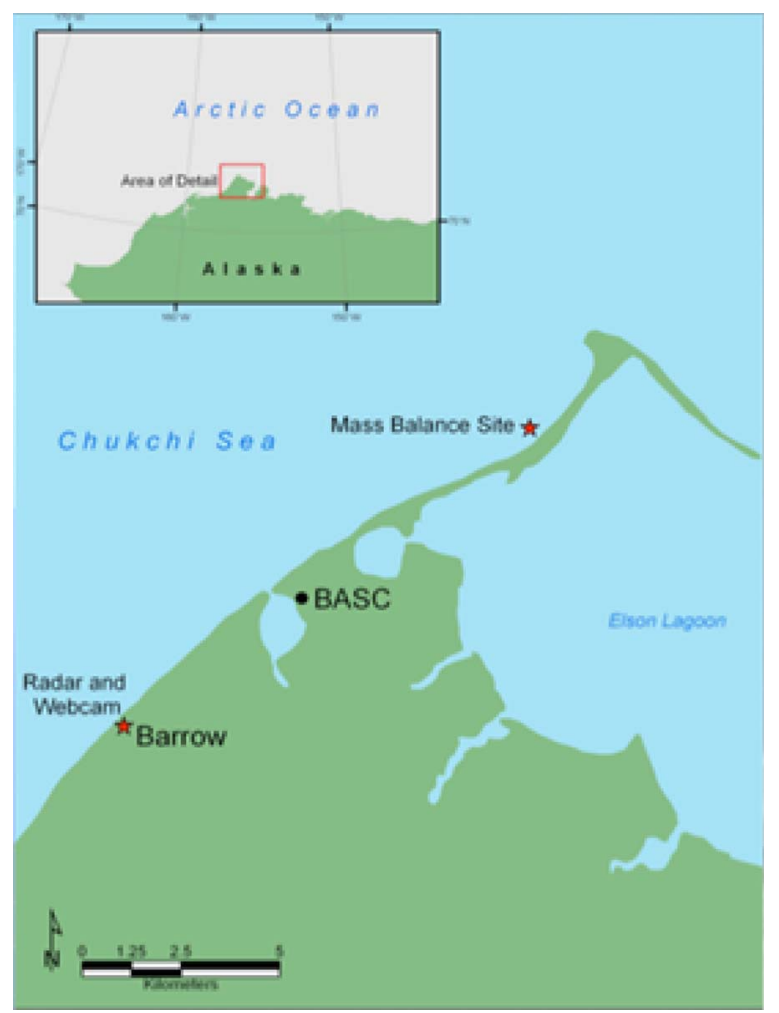

Fig. 1. Map of the study site and location of radar and other instrumentation.

TABLE I

Minimum Detectable Height of Reflectors for DIFFERENT RADAR FREQUENCIES

\begin{tabular}{|l|r|r|r|r|}
\hline Radar System / Range(km) & 1 & 5 & 10 & 20 \\
\hline Ku-band & 0.007 & 0.124 & 0.423 & 1.478 \\
\hline X-band & 0.010 & 0.164 & 0.557 & 1.910 \\
\hline
\end{tabular}

The work presented here builds on infrastructure put in place as part of the Barrow Sea Ice Observatory, a long-term effort to collect sea-ice data in the context of a changing Arctic and provide information on coastal ice conditions of value to local residents and other stakeholders [6]. Barrow, Alaska is the northernmost township in the United States, located on the shores of the Chukchi Sea, with nearby Point Barrow as the country's northernmost point bordering on the Beaufort Sea to the East (Fig. 1). Sea ice is a defining feature of the Chukchi Sea, which is ice-covered for much of the year. Landfast ice, attached to the coast and generally present from October through July, plays an important role in biological and geological processes, local Iñupiat culture and subsistence, and industrial activities [6]. Processes involving the landfast ice, such as formation, break-out events, and deformation, occur on timescales ranging from minutes to weeks. Remotely sensed data of the area typically lack the temporal and spatial resolution needed to track and potentially anticipate these processes and events. A land-based radar system allows nearcontinuous monitoring of ice conditions and events along the Barrow coastline. As part of the Barrow Sea Ice Observatory, we have deployed a land-based Furuno FR7112 10kW, $\mathrm{X}$-band $(3.0 \mathrm{~cm})$ marine radar for the purpose of observing coastal sea ice dynamics as well as surface vessels in the area. The radar system has an operational range of $11 \mathrm{~km}$, although 

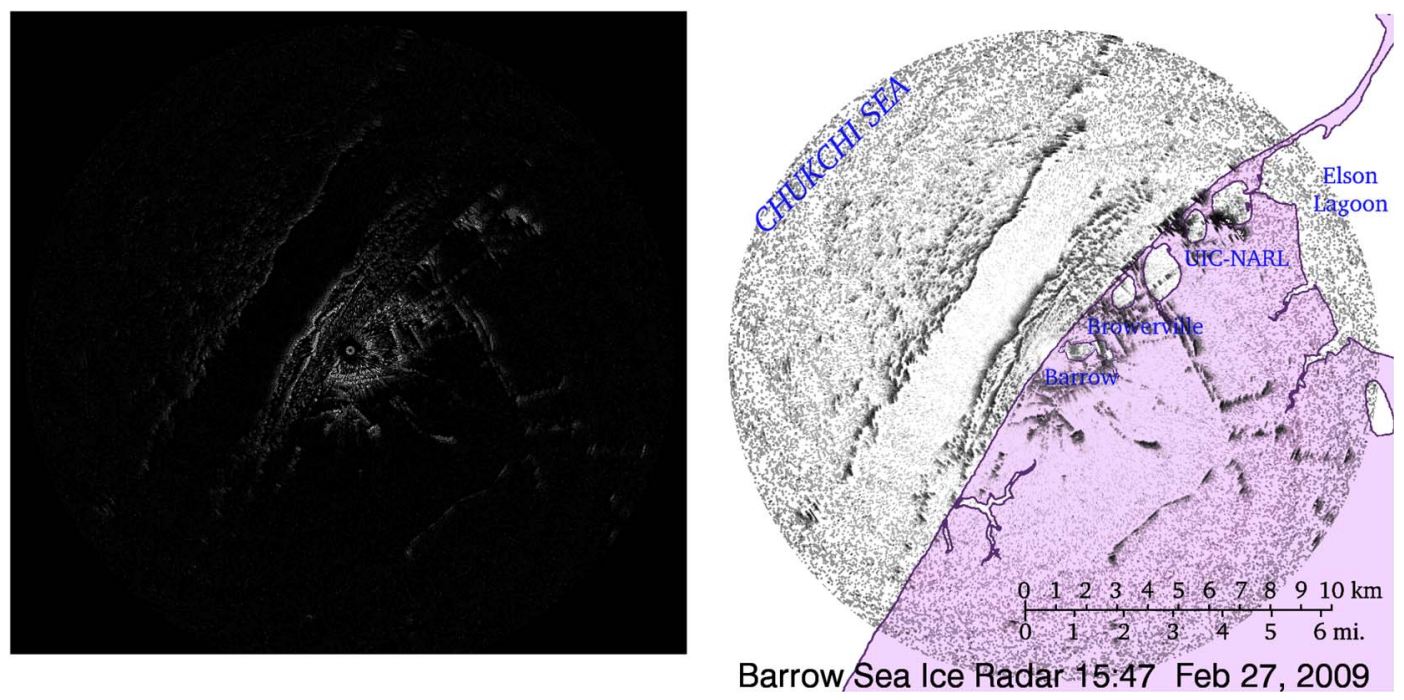

Fig. 2. (Left) An example of a radar scene. This image is one from a sequence used in training the analysis tools described below. (Right) Image data inverted for

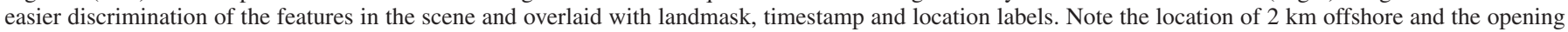
of a lead with the ice pack retreating toward the North (up).

the effective range varies with atmospheric and ice conditions. The radar's $1.65 \mathrm{~m}$ antenna provides a $1.2^{\circ}$ horizontal beamwidth and $22^{\circ}$ vertical beamwidth. Situated $22.5 \mathrm{~m}$ above sea level, the radar system achieves an azimuthal resolution of $209 \mathrm{~m}$ at a range of $10 \mathrm{~km}$, and a minimum detectable height of $0.34 \mathrm{~m}$ at $10 \mathrm{~km}$ range. The analog radar signal is digitized with the aid of a Xenex XN2000S radar controller/digitizer, putting out digital imagery that is posted online in near real-time which is archived.

$\mathrm{X}$-band radar has been chosen for the purpose of coastal sea ice monitoring over $\mathrm{Ku}$-band radars for a number of reasons, although primarily for the larger range capabilities in X-band. $\mathrm{Ku}$-band $(12-18 \mathrm{GHz}, 1.67-2.5 \mathrm{~cm})$ radar has a higher resolution than X-band and detects surface features more robustly. The increase in resolution comes with a loss in range and makes the radar system more susceptible to interference in ice detection by atmospheric effects and sea surface clutter [1]. Considering radar systems whose only difference is in the radar band used, such that other radar parameters like the radar wave pulse length, size of the antenna, height above sea level, and radio frequency power output are the same, the difference in minimum detectable height of a radar reflector amounts to only $0.134 \mathrm{~m}$ between $\mathrm{Ku}$ and $\mathrm{X}$-band at a range of $10 \mathrm{~km}$ (Table I), and decreases with decreasing range. Atmospheric attenuation of radar signals in polar regions is more pronounced at higher frequencies. The typical attenuation of $\mathrm{Ku}$-band radar signals is $0.09 \mathrm{~dB} / \mathrm{km}$, while the maximum can be up to $0.15 \mathrm{~dB} / \mathrm{km}$. The maximum attenuation of X-band radar is up to $0.01 \mathrm{~dB} / \mathrm{km}[1]$. Attenuation of all radar signals increases with the occurrence of precipitation and fog.

An example of a radar scene is shown on the left side of Fig. 2. This image is one from a sequence used in training the analysis tools described below. The panel on the right in Fig. 2 is based on the same image data, but has been inverted for easier discrimination of the features in the scene. A landmask, timestamp, and location labels have been applied for orientation. Near the shore of Barrow and surrounding areas, landfast ice is clearly visible, with linear features within the landfast ice being ridges formed in situ earlier in the ice season. The features in the upper left portion of the image correspond to ice floes part of the moving pack ice that is pulling away from shore toward the North (top of the image), leaving an area of open water and thin ice (lead) between the landfast ice and the ice pack.

\section{BACKGROUND: FEATURE SELECTION AND EXTRACTION}

Most areas in any image are either homogeneous or contain little information. Feature selection aims to select regions of an image that are relevant to a given task. In our problem, feature selection can help in accurate motion analysis of radar imagery and also track surface vessels. Below we first review some feature detectors used in the area of computer vision and then proceed to those used in radar imagery in particular.

Features are chosen in accordance with the interest strength associated with the region containing them. The interest strength is either assigned based on the autocorrelation on local patch such as in Harris corners [15] or through histogram of the orientations contained within a patch (for e.g., SIFT [16]). Other strength assignment schemes use discriminant information such as Fisher scores [17], maximization of marginal diversity (MMD) [18] and information theoritical measures [19].

For identifying and tracking targets in radar imagery, current approaches use characteristics of the radar signature [20], models of trajectory [21] and also scatter from between targets [22]. Yeary et al. [20] use an iterative particle filter approach for tracking of tornado related features. They use non-Gaussian Kalman filtering for estimating the trajectory of these features. Similarly, Weishi et al. [23] use Kalman filtering for tracking small moving targets in avian images. Other priors on trajectories include straight lines in 3-D space [24] and jump Markov model [21]. In our image sequences, we employed a constraint on the maximum displacement between frames and 
stronger priors were rendered infeasible due to complex motion of the individual reflectors. For a complete review of tracking techniques in radar imagery, we refer the reader to [3].

In the present paper, there are two kinds of features that are of interest in the radar imagery. One set of features concern the reflectors from ice and the other from surface vessels. We need to identify and track the ice reflectors to obtain an estimate of the motion field. Unlike the approaches discussed above, the features in the radar imagery need not be part of a single object or surface, hence identification of each feature point could be considered independent. The only criterion to be satisfied by the selected features is that they be amenable to tracking. In view of this, we use multiscale-Harris [25] detectors to identify the ice reflectors and track them using the Kanade-Lucas-Tomasi (KLT) tracker [26]. More details are provided in Section VI. To identify the surface vessels, we learn the appearance of regions containing vessels using manually identified training samples. Candidate regions in test images are identified using blob detection with Gaussian kernel. These regions are then compared with training patches with respect to principal components of intensity distribution to verify if the region represents a vessel. This approach is detailed in Section IX.

\section{BACKGROUND: ACTIVE CONTOURS AND DEFORMABLE MODELS}

We use the concept of active contours for delineating the edge between the moving sea ice and the stationary landfast ice. Active contours or snakes is one of the techniques in the larger framework of deformable models. For accurate segmentation over boundaries constituted by arbitrary curves/surfaces (no prior shape description), deformable models are used. They may be used to segment volumes [27], surface regions [28] or image regions [29]. We review the two major approaches to deformable models to motivate our approach.

Active contours may be solved using either parametric or geometric approach. The former uses a parametric model to define an initial curve. An external energy function (usually derived from image gradients) is designed to have low values along the desired boundary. An internal energy function is defined to regularize the contour by limiting its variation in space. The curve is then allowed to deform under a variational framework to obtain a contour that minimizes the internal and external energies. To make them more robust, some approaches define the external energy as the combination of image intensity and gradient [29]. On the other hand, geometric deformable models [30], [31] use the notion of level sets to solve the segmentation problem. The boundary of the shape is implicitly represented as the zero level set of higher-dimensional level set functions [32]. Instead of energy minimization, objective shape is recovered when the shape evolution velocity is reduced to zero. The advantages of geometric methods include ability to handle topological changes, differential geometric constraints and invariance of computational complexity with shape. However, most of these methods are aimed at closed curves/surfaces [33]. It is also difficult to enforce known topological constraints and they need a homogeneously evaluated level function (though a combination of level-set and variational framework has been proposed to handle sparse data [34]. In present work, we have a prior topology of the delineation and also the evaluation of the external energy is not from homogeneous data. We have thus opted for a parametric model of contour. The external energy is derived in terms of the observed velocity of pixels and the smoothness energy encodes the variations in the boundary. A contour is then deformed to pass through regions of low potential by minimizing the integral of energy in its path. The details of the method are provided in Section VII.

\section{Outline of Algorithms AND IMAGE-DERIVED PRODUCTS}

The radar imagery is derived with the aid of a Xenex XN2000S surveillance system (details in Section II) that has been programmed to provide raw range/backscatter data at 6-bit nominal dynamic range. The realized dynamic range of any given image is dependent upon atmospheric and ice condtions. When there is no reflector present at a location, the output is usually a noise signal whose magnitude is confined to the first three non-zero levels of output. The amount and amplitude seems to be range dependent with increased noise signals at further ranges from the center of the radar footprint. A greyscale image of size $1024 \times 1024$ pixels is acquired typically once every four minutes, though sampling is possible down to sub-minute intervals. Each pixel covers a rectangular region of $23.5 \mathrm{~m} \times 23.5 \mathrm{~m}$.

From these images, we obtain the following products:

1) Feature tracks-Tracking of reflectors robustly for the entire period that they are detectable in the imagery.

2) Motion field-Estimation of the average ice velocity field in the image, typically over a $24-\mathrm{h}$ period.

3) Location of landfast ice edge-Delineation of the edge of the stable landfast ice, typically derived from the daily ice motion fields.

4) Event detection-Detect environmental hazards and other anomalous events, such as landfast ice break-out events.

5) Surface vessel positions-Detect and locate surface vessels.

The workflow and products are summarized in Fig. 3. Preprocessing consists of the following. The image is first reduced to half the size in each dimension to reduce noise and also to speed up the processing by applying an average filter and then downsampling. Thus the images processed are $512 \times 512$ pixels with each pixel measuring $47 \mathrm{~m}$ in each direction. Images exhibiting large shifts due to errors in radar synchronization are excluded from processing. The derivation of motion fields is based on computations carried out on a 24-h image sequence. Both dense and sparse flow methods are used to obtain dislocation vectors for reflectors in all subregions of the image. An active contour is then used, with the average flow as the potential map, to obtain the boundary between the moving ice and the stationary ice.

The detection of anomalous or potentially hazardous events affecting subregions of the image builds on a Hidden Markov Model (HMM) approach for anomalous event detection, as developed by [35]. This method is particularly suitable as no motion or image segmentation is needed for its application. 


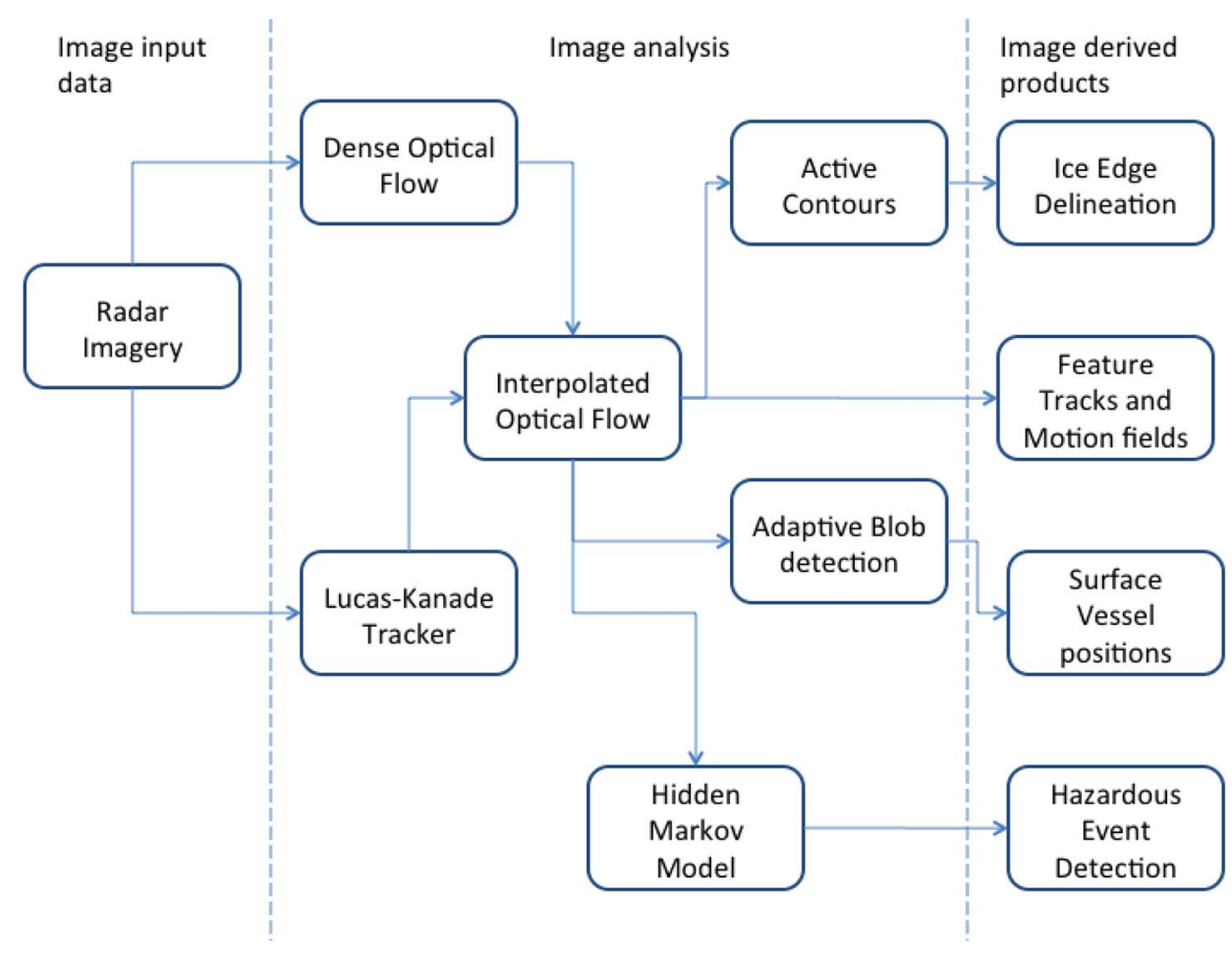

Fig. 3. Outline of our method and products.

The state of the observed ice, being a dynamic variable, has to be modeled by a continuous process. Thus, ice motion and deformation or changes in backscatter signal patterns are treated as stochastic processes whose properties can be modeled using HMM. The various phases in the motion are analogous to the symbols emitted by the HMM in the state transition. The model is trained using both positive (presence) and negative (absence) examples of hazardous events, in this case catastrophic ice break-out events. Surface vessels are detected using a bank of blob detectors and an appearance model obtained from training patches. These different methods and their implementation are discussed in detail in the following sections.

\section{Computation of Dislocation VECTORS AND MOTION FIELDS}

The derivation of a motion (or velocity) field of features in the imagery data is central to our application. Three of the image-derived products require derivation and analysis of a motion field (Fig. 3). Here, tracked features correspond to radar reflectors, which typically include ice features protruding above the surface of level ice, such as pressure ridges or small pieces of ice rubble oriented favorably to act as radar reflectors, as well as surface vessels and stationary infrastructure [1], [12]. The computation of motion fields using the radar ice imagery is rendered challenging by the nature of the data acquired. Typically, neither open water nor level ice are visible in the radar imagery. Pixels with non-zero grey values correspond to rough ice features or surface vessels. The aim is to obtain motion fields for the entire ice covered region. However, since the distribution of ice is not homogenous across the viewing area, and due to occlusions, large regions of the image may appear without any backscatter signals most of the time (as

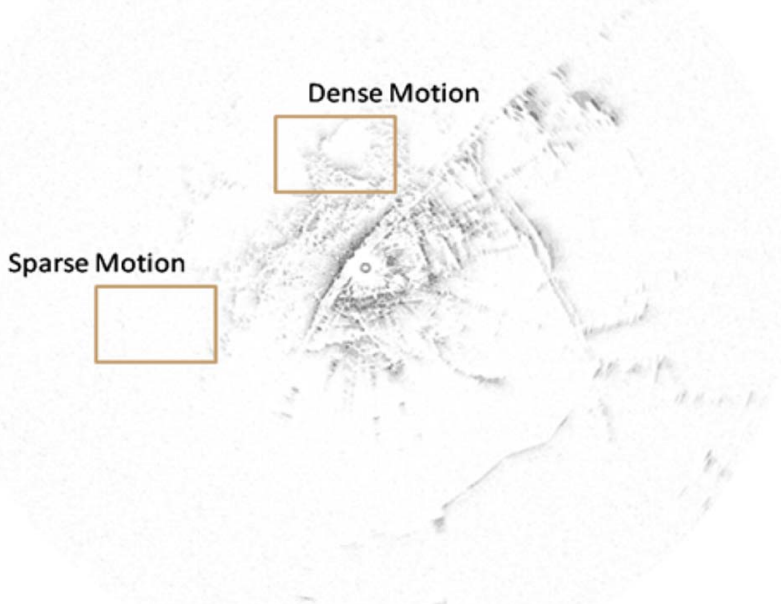

Barrow Sea Ice Radar 17:46 Dec 10, 2007

Fig. 4. Sample image showing regions of dense and sparse flows. Regions with large number of moving reflectors can be identified with dense motion detection techniques whereas regions which contain only few moving reflectors will appear stationary in these methods. Sparse motion estimation methods such as feature tracking are useful in this case.

seen in Fig. 4 in the region shown at left). A traditional motion estimation algorithm, such as dense optical flow techniques, would report these regions as being stationary. At the same time, ice features may only generate radar reflections for part of the time because of changes in reflector orientation or surface state. For the purpose of identifying boundaries between stationary ice and ice at motion as well as for derivations of ice kinematic and deformation fields, a more accurate motion field is needed at high spatial resolution. To solve this problem, we employ both dense and sparse optical flow methods. The 
dense method works where more reflectors are visible, and the sparse feature tracking method obtains the motion field for other subregions of the image. We employ a Normalized Cross Correlation $(\mathrm{NCC})$ approach within windows of size $5 \times 5$ pixels to derive dense motion estimates $\left(V_{d}\right)$. In areas with few reflectors, this approach is prone to errors due to the presence of spurious backscatter or image noise. Hence, we suppress the dense motion estimates when the ratio of errors between the first and the next best match is lesser than a preset threshold (this is learned using manually selected sample patches in training images). To minimize errors, the search range for dense motion estimate is restricted based on the maximal observed flow. Overlapping windows are used in cases where the motion field needs to be densely sampled.

\section{A. Tracking Features for Motion Field}

To detect the features in the radar image, we need to identify regions of the image which contain reflectors that can be easily tracked across frames. Though some of the reflectors may correspond to a single physical object at sea, grouping them is rendered difficult due to the complex nature of occlusion and frequent non-rigid transformations (breaking, collisions). Thus, we attempt to identify and track individual reflectors independently. Owing to the nature of radar imagery, attributes such as image gradients are prone to error and hence orientation based detectors [16] are not suitable. We identify features using the multiscale Harris [25] operator as those regions in the image which have a corner-like appearance. It has been shown to identify features which can be reliably tracked under motion. This approach uses the correlation between an image patch and its neighboring region to identify the uniqueness of the patch. The sum of intensity squared difference errors $(S)$ between a region of image $I$ and a patch obtained by a window shifted by $(x, y)$ can be assumed to be bilinear in the window shift

$$
S(x, y)=\left[\begin{array}{ll}
x & y
\end{array}\right] A\left[\begin{array}{ll}
x & y
\end{array}\right]^{T} .
$$

The eigenvalues of $A$ provide an indication of the uniqueness of the patch with respect to its neighbors. If both the eigenvalues are small, it indicates that the patch is homogenous. If only one of the values is very small then it indicates that the region contains an edge. However, if both the values are large, then it indicates that the region has a corner-like appearance. Such analysis of eigenvalues is repeated over different scales of the image and only those regions which are consistently prominent features at different scales are chosen for tracking. Since the density of reflectors may vary due to the nature of prevalent flow, we do not suppress any features.

Usually, the detected features are described using feature descriptors based on the appearance of the feature. Such descriptors of features found in multiple frames are compared to establish correspondences. In our case however, since the gray value of a reflector is sensitive to the orientation of the radar reflector, the appearance of features in individual radar scenes changes very rapidly and very few features can be tracked over more than 10 frames (i.e., 40 minutes). Also, many of the features are similar in appearance (especially when they appear in the far field of the radar). Thus a descriptor based approach to establish correspondences among features of different frames is not feasible. Instead we rely on the dense temporal sampling to track the features locally. We employ the KLT tracker approach [26] to track features (i.e., persistent radar reflectors). The method makes following assumptions about the feature tracks: the flow is constant in a window around the feature point and the difference in image intensity due to motion can be expressed as a linear function in time. Both these conditions are easily met in the image sequences we explore. Since it enforces a linearity in image differences and performs localized search for the location of feature, the constraints imposed on motion are stronger than those assumed in a constant velocity tracks with Gaussian noise (e.g., Kalman filters). If $I_{x}, I_{y}$ and $I_{t}$ represent the horizontal, vertical and temporal derivatives in image intensity around a feature point in the image, the vertical and horizontal velocities ( $v_{x}$ and $v_{y}$ ) are given as a solution to the linear system below. The summation is over all the pixels $p$ in the chosen window around the feature

$$
\begin{aligned}
\left(\begin{array}{cc}
\sum_{p} I_{x}(p)^{2} & \sum_{p} I_{x}(p) I_{y}(p) \\
\sum_{p} I_{x}(p) I_{y}(p) & \sum_{p} I_{y}(p)^{2}
\end{array}\right)\left(\begin{array}{c}
v_{x} \\
v_{y}
\end{array}\right) & \\
= & \left(\begin{array}{l}
-\sum_{p} I_{x}(p) I_{t}(p) \\
-\sum_{p} I_{y}(p) I_{t}(p)
\end{array}\right)
\end{aligned}
$$

The intensity of the windows are premultiplied by a Gaussian centered at the feature location to decrease the contribution of pixels at the boundaries of the window (to compensate for the constant flow assumption). We are only interested in the average motion field at a given position of the image. We thus associate the velocities of the feature points with their initial position. To avoid generating false tracks for reflectors that flicker, only those features have been tracked that appear in more than 3 frames. Once the velocity field has been compiled from dislocation vectors derived for all features detected during a single day, a dense motion field $(V)$ over the image is obtained by linear interpolation from those points. Before the interpolation, the sparse velocities which differs from the dense estimate by a fixed threshold are removed.

\section{Delineation of Stable ICE}

We use parametric deformable models to estimate the contour that delineates stable ice. In such a model, shape is represented as an active parametric curve and associated with some energy values. Energy values are calculated by combining the external forces, which push the active curve toward salient image features such as edges, and internal forces, which constrain the curve smoothness. From some starting position, the active curve deforms itself toward desired image features while its associated energies are minimized. In the original parametric deformable model [36], the active curve is defined as a continuous spline $\mathrm{v}(\mathrm{t})=(\mathrm{x}(\mathrm{t}) ; \mathrm{y}(\mathrm{t}))$ with the curve arc length, $s$, as the parameter. The shape of the active curve is constrained by defining the curve's internal energy as the summation of magnitudes of the curve's first- and secondorder derivatives with respect to the curve arc length. Minimization of the first-order derivative forces the curve to be continuous. Curve smoothness is guaranteed by minimizing the 

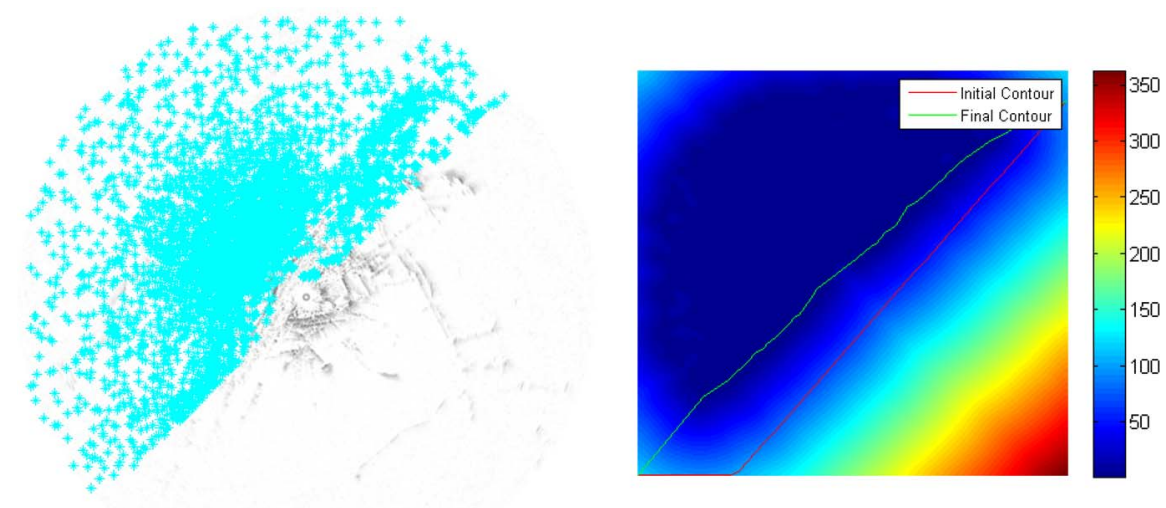

Barrow Sea Ice Radar 18:47 Feb 27, 2009

Fig. 5. (Left) Mobile points tracked in the image sequence over a period of about $4 \mathrm{~h}$. (Right) Potential derived from the mobile points represented as colored

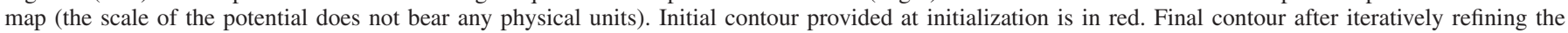
initialized contour shown in green.

second-order derivative so that there are no abrupt shape changes along the curve. The active curve is attached to the image by external energy

$$
E_{e x t}=\int|\nabla I(v(t))|^{2}
$$

where $I(v(t))$ is the image intensity value and $\nabla I(v(t))$ is the image gradient at the position where $v(t)$ is located. Since image gradient values are high at edges, minimization of the external energy pushes the active curve toward edges. Optimal position of the active curve in the image is decided by solving a set of partial differential equations whose solution gives the minimization of the curve energy.

Since we are interested in segmenting the stationary regions of the image, we use the motion of the coastal ice to estimate the external energy. However, an accurate dense estimate of motion is not easily obtained in all regions of the image due to the sparse nature of the radar reflectors. Using a window based dense motion estimate will bias the energy map leading to inaccurate delineation. A method of interpolating the observed sparse motion to an energy value at every pixel of the image is needed. We propose a method for deriving such a map based on a binary classification of tracked features. Each feature tracked in the image is labeled mobile or stationary based on the magnitude of the velocity at that point. Several reflectors that are physically stationary appear to undergo minute oscillations due to change in atmospheric conditions or radar noise. Using only inter-frame motion estimate will incorrectly label these reflectors as mobile. We propose to use the dense flow estimate and gross motion observed among multiple frames to filter such reflectors. The criteria for a feature to be mobile are that it conforms to the dense flow estimate for that frame and that its velocity is at least 100 pixels in 70 frames. The sum of mobile points (features detected in a radar backscatter image) tracked over the course of around 70 frames that is around $4 \mathrm{~h}$ is shown in Fig. 5 (left). The delineation of stable (i.e., stationary) landfast ice is carried out by employing the concept of active contours. A potential map, $P$, is derived using the distance transform of the mobile points. The distance transform at a given point indicates the distance of that point to the closest mobile point. Thus the distance transform at a mobile point would be zero and that at the lower right corner of the image would be highest (due to the orientation of our imagery).

The potential map and the initialization with a straight line contour is shown in Fig. 5 right. Since we know that the delineation contour is a simply connected open curve, the contour is parameterized by $t$ that lies between 0 and 1 , and the points on the contour are $(x(t), y(t))$. Using a level set approach in this case would lead to detection of multiple segmentations as smaller empty regions (without reflectors) may exist in the region corresponding to open sea. Instead, we seek a solution where the energy, $E$, on the active contour is minimized. This energy term is derived out of all the moving features on a single day, as we are interested in daily edge predictions. We should note that if a feature is mobile during any part of the day in a certain region, that region would not be within the landfast boundary (will not be considered stationary). The component $E_{p}$ accounts for the potential and $E_{C}$ accounts for the smoothness of the contour. The line is divided into segments and the endpoints are iteratively refined (using the Euler-Lagrange equations derived from the integral) until the energy is at minimum

$$
\begin{aligned}
E & =\int_{l} E_{P}+\alpha E_{C} \\
E_{P} & =-|\nabla P|^{2} \\
E_{C} & =-\left|x_{t}\right|^{2}-\left|y_{t}\right|^{2} .
\end{aligned}
$$

The final contour, produced after the convergence of the above optimization process, will provide the estimate of the ice edge boundary. The initialization contour is always defined in the stationary part of the image (corresponding to land), so that it can then be stretched by the gradient in the potential field toward the stable ice boundary. The weight for the smoothness term is chosen based on evaluations performed on sample image sequences and the value is fixed for the cases where error analysis is carried out. The initial contour is represented using 50 equally spaced points. The energy function is minimized using line search gradient descent. Alternatively, dynamic programming techniques suggested by Amini et al. [37] may be 


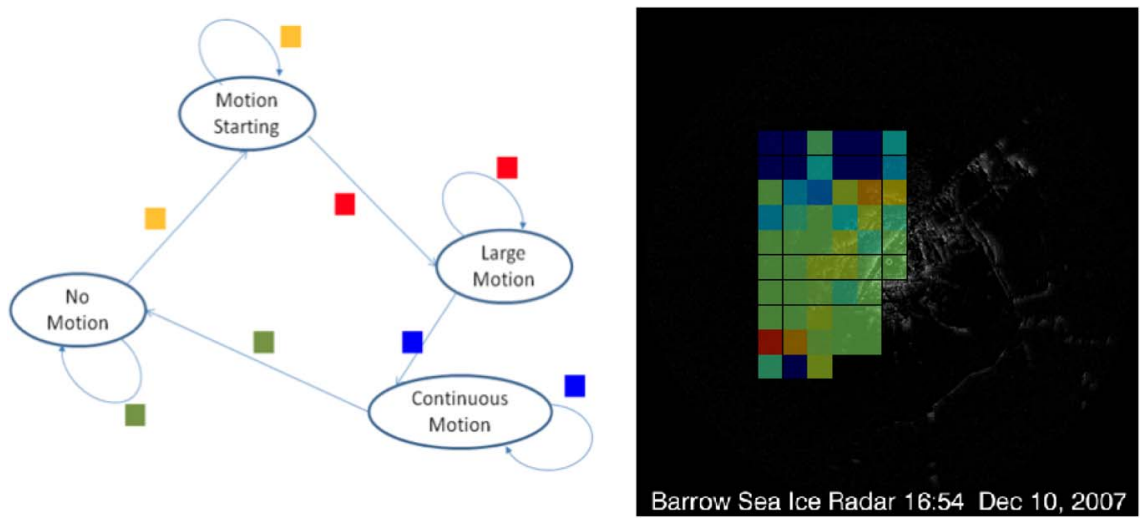

Fig. 6. (Left) Schematic showing four different ice states and their expression in the form of indicators (shown as colored symbols) as estimated by an HMM for break-out events. (Right) Grid points labeled (colors correspond to those shown at right).

used. Dynamic programming separates the optimization process into independent stages involving local neighborhood of points.

\section{Detection of ICE HAZARd Events}

We employed a Gaussian Mixture Hidden Markov model (GM-HMM) to automatically determine and track potentially hazardous events, in particular break-out events that remove part of the stable ice cover (developed by Porikli and Li [35] to characterize vehicle traffic conditions). The goal of this work is to identify such events in their earliest stages to allow for effective on-ice response. Furthermore, we want to explore whether such events are associated with potential precursors [see also [12]] that may help in anticipating break-outs and other anomalous events. An input vector (q) for each box of size $25 \times 25$ as shown in Fig. 6 (right) was generated using the motion field described above $-\left[v_{x}, v_{y}, d\right]$ where $v_{x}, v_{y}$ is the motion vector and $\mathrm{d}$ is the estimated divergence in the box. The states (s1, s2, s3, s4) correspond to:

1) No motion (stable landfast ice)

2) Incipient motion (potential precursor to break-out event)

3) Substantial, anomalous motion (potential break-out event in progress)

4) Steady, ice drift motion (movement of ice associated with steady offshore drift)

The states are shown in the left panel in Fig. 6. The indicators that are being tracked as expressions of underlying ("hidden") characteristics of the flow field are shown as colorcoded symbols, with different colors indicative of different transitions between states. The probabilities of the observed velocity and divergences in each state are assumed to be normally distributed around a number of modes. Hence, each state, $s_{i}$, is characterized by a mixture of 3-D Gaussians corresponding to the velocity and the divergence. Hence, each Gaussian, j, belonging to the $i$ th state, $G_{i j}$, is composed of a vector of means, $\mu_{i j}$, and a covariance matrix, $\Sigma_{i j}$. The observation probability that a certain input vector $q$ in Gaussian $j$ of state $i$ is given by the following:

$$
p_{i j}(q)=\frac{1}{2 \pi \operatorname{det}\left(\Sigma_{i j}\right)^{2}} e^{\frac{-\left(q-\mu_{i j}\right)^{T} \Sigma^{-1}\left(q-\mu_{i j}\right)}{2}} .
$$

The transition matrix A describes the probability of transition from one state to another

$$
A=\left(\begin{array}{cccc}
a_{11} & a_{12} & \cdots & a_{14} \\
a_{21} & a_{22} & \cdots & a_{24} \\
\vdots & \vdots & \ddots & \vdots \\
a_{41} & a_{42} & \cdots & a_{44}
\end{array}\right)
$$

For example, $a_{31}$ represents the probability that the previous state was 3 and the next state is 1 . The parameters that are to be estimated are grouped into one as $\lambda=[A, \mu, \Sigma]$. The aim of parameter learning is to find

$$
\lambda=\operatorname{argmax}(\log (p(v \mid \lambda))) .
$$

Once the parameters are estimated, the states corresponding to an arbitrary input vector sequence can be decoded using the Viterbi algorithm [38].

Radar imagery obtained at four minute intervals for three consecutive ice seasons starting in fall of 2006 was analyzed manually to identify all break-out events that occurred during this time interval. The sequences of radar imagery were compiled into animations that covered 10 days. An operator familiar with the radar system, the Barrow area, and the nearshore ice processes visible in the radar imagery analyzed the animations. The initial appearance of ice in the radar footprint, the date of first stationary ice, accretion and removal of landfast ice, deformation events, occurrence and severity of break-out events, and phenomena visible in the radar, such as atmospheric effects and reflector strength variations, were documented. The image sequences were then partitioned into time periods with a break-up event and those without. The individual images were grouped into two sets, one to train the model (estimating the parameters for each state in the Markov model) and the other to test model performance. Each set consisted of the four positive and negative examples. These were used to train a GM-HMM with four different ice states. In each state, the probability distribution of the observed variables consisted of a combination of four Gaussians. The initial means and covariances were distributed uniformly based on the observed examples. HMM was trained using the Baum Welch Expectation-Maximization approach available in Matlab. As shown in Fig. 5, the predicted states were then color-coded to highlight potentially unstable 
ice regions (shown in orange and red). A break-up event was classified based on at least eight adjacent cells exhibiting substantial anomalous motion.

\section{Identification of SuRface Vessels}

The sampling rate of the current radar configuration (one scene every four minutes) does not provide data of sufficient temporal resolution to generate tracks for typical surface vessels using the motion tracking algorithms described above. While this will be improved in future work, surface vessels may be identified based on their radar signatures, as practiced in common radar target identification approaches [3]. However, the challenge in ice-covered regions is that ice features typically appear quite similar to artificial structures. To address this challenge, vessel identification was based on determination of the characteristic shape and brightness profile of the vessels using principal component analysis techniques.

The identification of surface vessels is carried out in two steps: selection of candidate regions and filtering based on appearance model. The candidate regions are selected using a blob-detector with Gaussian kernel. The radar image is convolved with demeaned, normalized Gaussian kernels with various bandwidths and window sizes. The kernel $\tilde{G}$ with a window size of $W$ and a bandwidth of $\sigma$ centered around $\left(x_{0}, y_{0}\right)$ is defined as follows:

$$
\begin{aligned}
G\left(x, y ; x_{0}, y_{0}, \sigma\right)= & \frac{1}{2 \pi \sigma^{2}} e^{-\frac{\left(x-x_{0}\right)^{2}+\left(y-y_{0}\right)^{2}}{2 \sigma^{2}}} \\
\tilde{G}\left(x, y ; x_{0}, y_{0}, \sigma, W\right)= & G\left(x, y ; x_{0}, y_{0}, \sigma\right) \\
& -\sum_{\left(x_{1}, y_{1}\right) \in W} G\left(x_{1}, y_{1} ; x_{0}, y_{0}, \sigma\right) .
\end{aligned}
$$

The convolution kernel $\tilde{G}$ contains both negative and positive values which sum up to zero. If the kernel is applied to a homogeneous image region, the response will be zero as it will just be a scaled sum of all the entries in the kernel matrix. Also, regions which are entirely dissimilar to the kernel will have low positive or large negative value. Large positive response is obtained only in the regions of the image that contained intensity characteristics similar to a specific kernel. Using manually selected patches that contain surface vessels, the bandwidths and window sizes that are most suitable for vessel detection are learnt. Though the candidate patches from this step identified most of the surface vessels, some large reflectors from ice were also selected. To filter out such reflectors, filtering based on appearance model is performed.

High-backscatter image features corresponding to surface vessels were manually selected. The pixel values in each patch were then arranged in a column vector and these arranged in a matrix. The principal components of this matrix were extracted through eigenvalue decomposition. For each test image, candidate features were first detected using a bank of Gaussian filters. These features were then compared with the principal components derived from positively identified vessels. If the residual of this comparison was greater than a fixed threshold, the features were classified as ice (or non-vessel) and discarded, else they were classified as a surface vessel and retained. Exam-

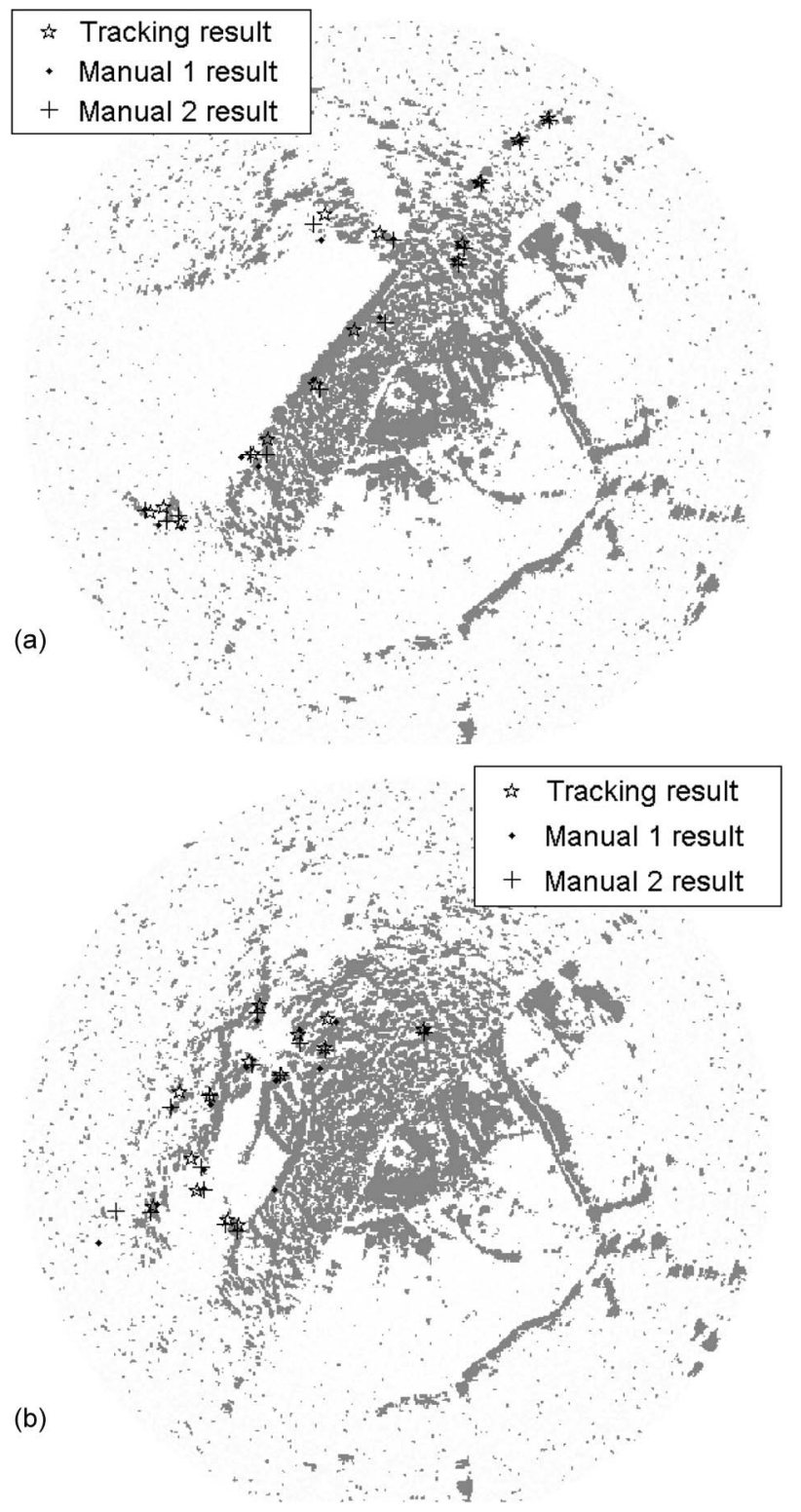

Fig. 7. Comparison of manually and automatically tracked ice features to derive velocity fields. Sample frames from two days are shown above (the gray areas indicate radar returns): (a) January 8, 2008, 2:43 local time, (b) January 6, 2008, 2:18 local time.

ples of vessel patches with the extracted appearance model can be seen in Fig. 11. It was verified that the principal components chosen were able to explain most of the variation present in the training patches. Details of validation are provided in the Section X-D.

\section{Application, Validation, and Results}

\section{A. Feature Tracking}

Feature tracking to derive ice velocity fields was performed using the methods described in the previous sections. To assess the accuracy of the method, the performance of the automated algorithm was compared against manual tracking of a large set of randomly assigned feature points by two expert operators (Fig. 7). This was achieved by displaying the initial and final 


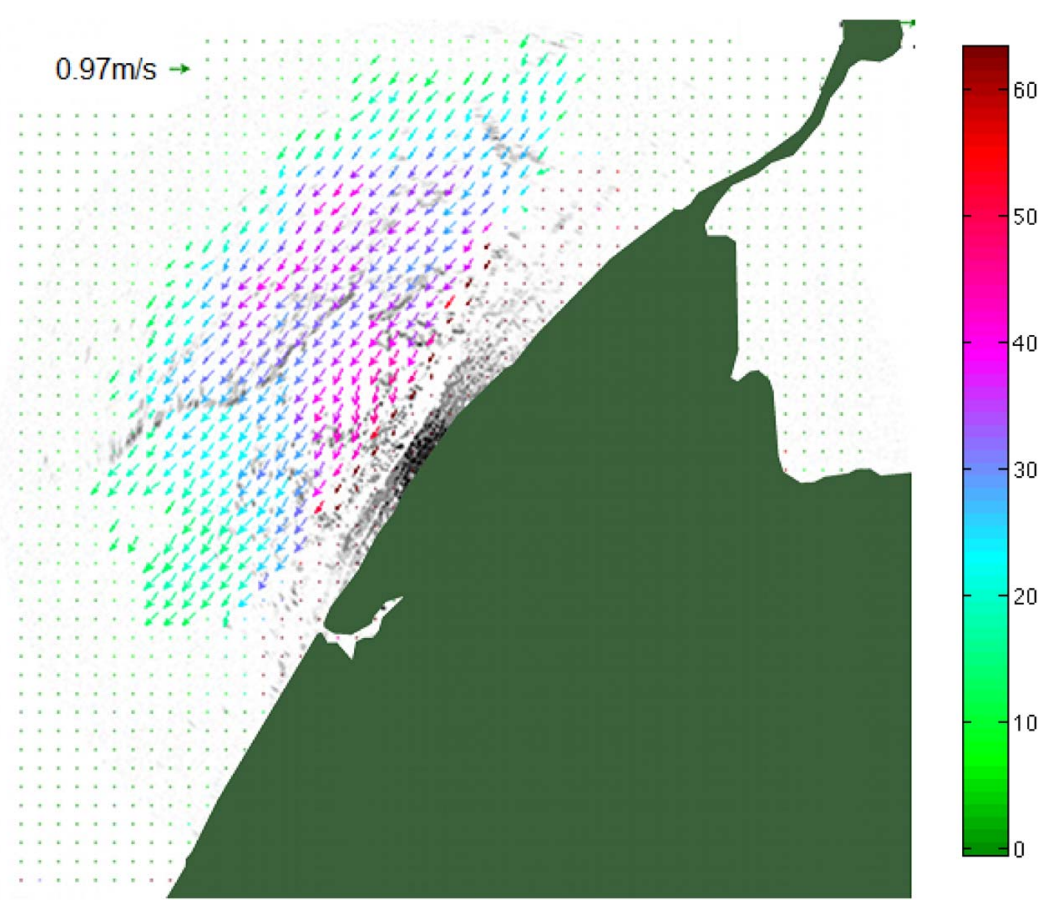

Fig. 8. Velocity field derived from February 3, 2010 radar imagery. The length and direction of the arrows corresponds to the estimated mean velocity for each grid cell, whereas the color indicates the number of frames over which the average is calculated. The flow is filtered using a mask based on the number of frames for which features were successfully tracked in each cell $(N \leq 20)$.

position of tracked features in two separate images. The final position of the tracked feature was then offset by adding Gaussian noise to the $x-y$ location of the tracked feature. The user was then asked to shift this marker to the correct final position corresponding to the feature identified in the initial frame of the sequence.

The error statistics computed from these intercomparisons between manually and automatically determined dislocation vectors are summarized in Table I and indicate that that errors are within acceptable limits. The evaluation was performed on two data sets with two independent manual evaluations per data set. Set 1 consisted mostly of points on moving ice whereas set 2 included some points on the static ice as well. Fig. 7 shows the comparison of manually and automatically tracked points for two image sequences. In summary, for features trackable across much of the image, the corresponding error in the dislocation vector is on average well below $10 \%$ of the tracked length. Since these are zero-mean errors, velocity fields obtained for daily or longer intervals are much smaller and associated with errors of a few percent at most.

An example for an ice velocity field, derived from the feature-tracking program, is shown in Fig. 8 for February 23, 2010. The velocity field fully captures offshore ice pack motion, with large ice floes moving alongshore toward the southwest throughout the day. The length and direction of the arrows corresponds to the estimated mean velocity for each grid cell, whereas the color indicates the number of frames over which the average is calculated. Some of the large floes were seen rotating counterclockwise as they moved through the radars field of view. These aspects of ice motion and consistent rotation (likely driven by interaction with the topography and persistent current patterns) are reproduced in the vector field.
The landfast ice shows little to no motion; with small velocity magnitudes likely due to fluctuations in signal intensity or in reflector orientation relative to the radar unit due to atmospheric conditions or oceanic processes, such as changes in sea level. Note that the radar mask is determined by the number of frames for which features were successfully tracked in each cell $(N \leq 20)$, with data below this threshold excluded from the analysis. Some of the velocity vectors at the outer margin of the mask may be associated with larger errors than those for the bulk of the area. However, the cutoff criterion can be adjusted to improve discrimination and reduce errors based on sea ice conditions and the physical process under investigation.

The ice velocities determined from the radar imagery have been compared to ice velocities determined from satellite imagery. Specifically, the maximum averaged daily ice velocity on February 3, 2010 was $0.97 \mathrm{~m} / \mathrm{s}$ (Fig. 8), while the average for a grid cell proximal and partially overlapping the radar footprint was about $0.1 \mathrm{~m} / \mathrm{s}$ as determined from the Advanced Microwave Scanning Radiometer-Earth Observing System (AMSR-E). Both approaches are consistent in indicating ice movement to the Southwest. The method for determining ice velocity from AMSR-E imagery is comparable to the maximum cross correlation methods for deriving sea ice velocity fields from Scanning Multichannel Microwave Radiometer (SMMR), Special Sensor Microwave/Imager (SSM/I), Advanced Very High Resolution Radiometer, and International Arctic Buoy Program (IABP) data outlined in [39], ([40]). Differences in the magnitude of ice velocities derived from satellite imagery and ground-based radar systems may arise from the resolution differences of the two tracking methods and the fact that passive microwave data are associated with potentially large errors in grid cells adjacent to land. The AMSR-E data, acquired at a spatial scale 
TABLE II

ERROR STATISTICS FOR POINT TRACKING

\begin{tabular}{|l|l|l|l|}
\hline Data & Error Mean (in pixels) & Error Median (in pixels) & Error Variance (in pixels) \\
\hline Manual Trial 1-Set 1 & 2.75 & 2.67 & 1.93 \\
\hline Manual Trial 2-Set 1 & 2.22 & 2.17 & 1.70 \\
\hline Manual Trial -Set 2 & 0.35 & 0.15 & 0.47 \\
\hline
\end{tabular}

TABLE III

ICE EDGE DELINEATION ERRORS

\begin{tabular}{|l|l|l|}
\hline Date & Error Mean (in pixels) & Error Mode (in pixels) \\
\hline 20 Jan 2008 & 11.18 & 5.09 \\
\hline 22 Jan 2008 & 9.988 & 1.00 \\
\hline 03 Apr 2008 & 6.393 & 1.00 \\
\hline 07 Apr 2008 & 5.164 & 3.60 \\
\hline 27 Apr 2008 & 5.518 & 1.00 \\
\hline 15 Nov 2008 & 4.48 & 2.82 \\
\hline 27 Nov 2008 & 6.125 & 2.828 \\
\hline
\end{tabular}

of approximately $12.5 \mathrm{~km}$ by $12.5 \mathrm{~km}$, is reprojected onto a $25 \mathrm{~km}$ by $25 \mathrm{~km}$ grid, which is much larger than the area of the $10 \mathrm{~km}$ radius radar footprint. Hence, satellite-derived velocities are determined over neighborhoods of grid cells much larger than even the largest ice floes tracked by the coastal radar. Due to high gradients in ice velocity and short-term variability in ice velocity at time scales sampled by the radar, but not the satellite data, such large differences between higher radarderived velocities and lower satellite-derived velocities are to be expected. Moreover, small ice floes in what has become known as northern Alaskas Chukchi flaw zone, separating the stationary coastal ice and the offshore ice pack, can move at substantially higher velocities than the ice pack, sometimes more than an order of magnitudes faster ([41]).

\section{B. Landfast Ice Edge Delineation}

The landfast ice edges generated with the approach outlined above, follow the boundary between moving and stationary ice closely, even in regions toward the margins of the radar footprint where few features are trackable. To validate the results obtained with the automated approach, we traced the landfast ice boundaries manually in several radar-image sequences. To compare these boundaries with the automatically traced edges, a distance map that indicates the distance between a given pixel and the closest point on the automatically determined edge was derived. From this map we calculated the mean and mode of the distances along the manually marked edge traced through the distance map. This yields an unbiased estimate of the difference between the two curves. The errors observed in some cases are shown in Tables II and III.

To further explore the source and magnitude of these errors in derived landfast ice edges, an example of manually and automatically determined landfast sea ice edges is shown in Fig. 9. These scenes were recorded in 2007 on May 27 (a), 28 (b), and 29 (c), before, during, and after a late-season landfast ice breakout. This break-out, occurring at a time where just prior a large number of people from the community of Barrow had been on the ice, represented a major hazard [6]. For comparison, we also show the landfast ice edge derived from RADARSAT synthetic aperature radar (SAR) imagery, employing the method devel- oped by Mahoney et al. [42] (Fig. 9). In Fig. 9(a), the programgenerated ice edge is shoreward of the manually determined ice edge. This may be due to the nature of the stationary reflectors used to manually determine the ice edge, which varied in intensity due to changes in backscatter strength throughout the day of May 27. The program that determines the seaward landfast ice edge finds the average edge throughout any given day, such that variations in reflector intensity may increase errors in the derivation of velocity vectors and assign non-stationary ice labels to these landfast ice edge points. Comparison with imagery collected in February and March which lacks such offsets, suggests that this error is mostly due to variations in backscatter strength as a result of melt-freeze cycles in late spring.

Aliasing effects due to the daily averaging of velocity fields used in determining the ice edge explains the seaward location of the automatically determined ice edge in Fig. 9(b). The break-out occurred on this day; therefore the programgenerated ice edge reflects some intermediate state between the more seaward edge in the morning and the resulting shoreward edge at the end of the day. The discrepancies between the different ice edges depicted in Fig. 9(c) are likely due to a number of different factors. The program-generated edge, from northwest of the image center extending to the southwest, may be capturing occlusions that are possibly the result of the very strong, linear reflectors that define that section of the manually determined ice edge, which may be too close to shore. It is not uncommon to find areas of thin, stationary ice beyond the reflectors apparent in Fig. 9(c) and the automated algorithm may capture the stationary nature of this ice through the daily averaging. Part of the deviation between these ice edge metrics is due to the intermittent nature of ice motion along the edge, which enters into the daily mean velocity field but not the instantaneous manual outlining of the ice edge. The northern section of the manually determined edge is more seaward than the program generated edge most likely for the same reason as that of Fig. 9(a), with varying reflector backscatter intensity increasing the error in derived velocities.

The landfast ice edge in Fig. 9(c) determined from satellite imagery corresponds to the minimum ice extent observed for a 21-day period, or three consecutive radar mosaics of Alaskas northern coast. Here, the 21-day period spans from May 20 through June 10. Therefore, this edge also shows further ice edge retreat after the break-out event on May 28, although no significant ice edge changes occurred after the break-out. The step-like shape of the satellite-derived edge is due to the resolution differences between the radar and satellite imagery. The overestimation of the manually determined ice edge relative to the satellite-derived edge may also be due to the nature of the radar imagery and methods used in manually determining the edge, where the most seaward stationary radar returns are 
(a)

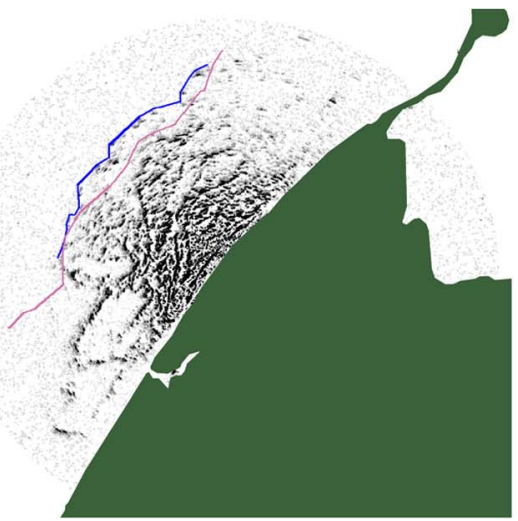

(b)

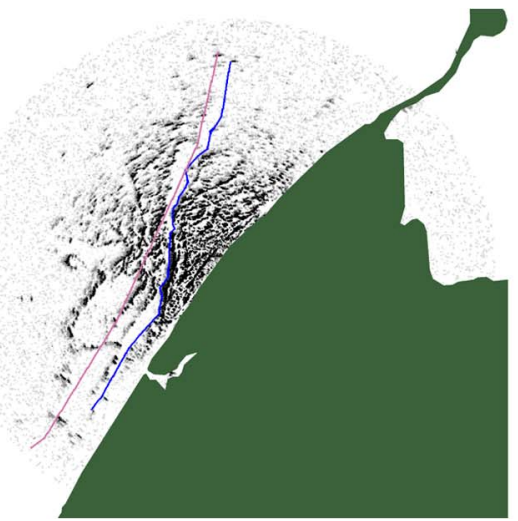

(c)

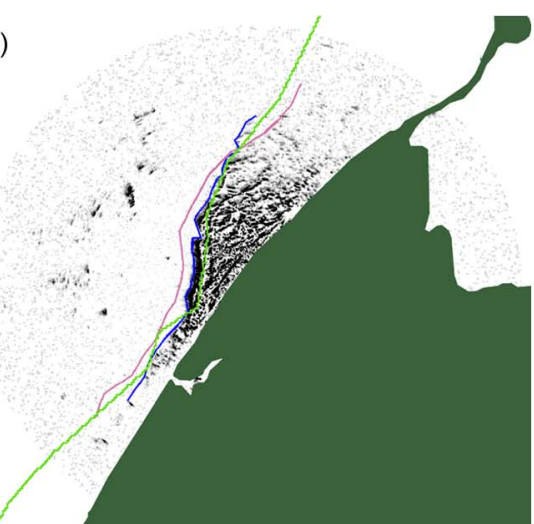

Fig. 9. Radar images (the black dots indicate radar returns) with manually determined landfast sea ice edge (blue), landfast ice edges derived with the automated approach (purple), and, in (c), the landfast ice edge determined from RADARSAT imagery (green). (a) Pre-break-out image from May 27, 2007. (b) Image from immediately after break-out event on May 28, 2007. (c) Post-break-out image from May, 29, 2007.
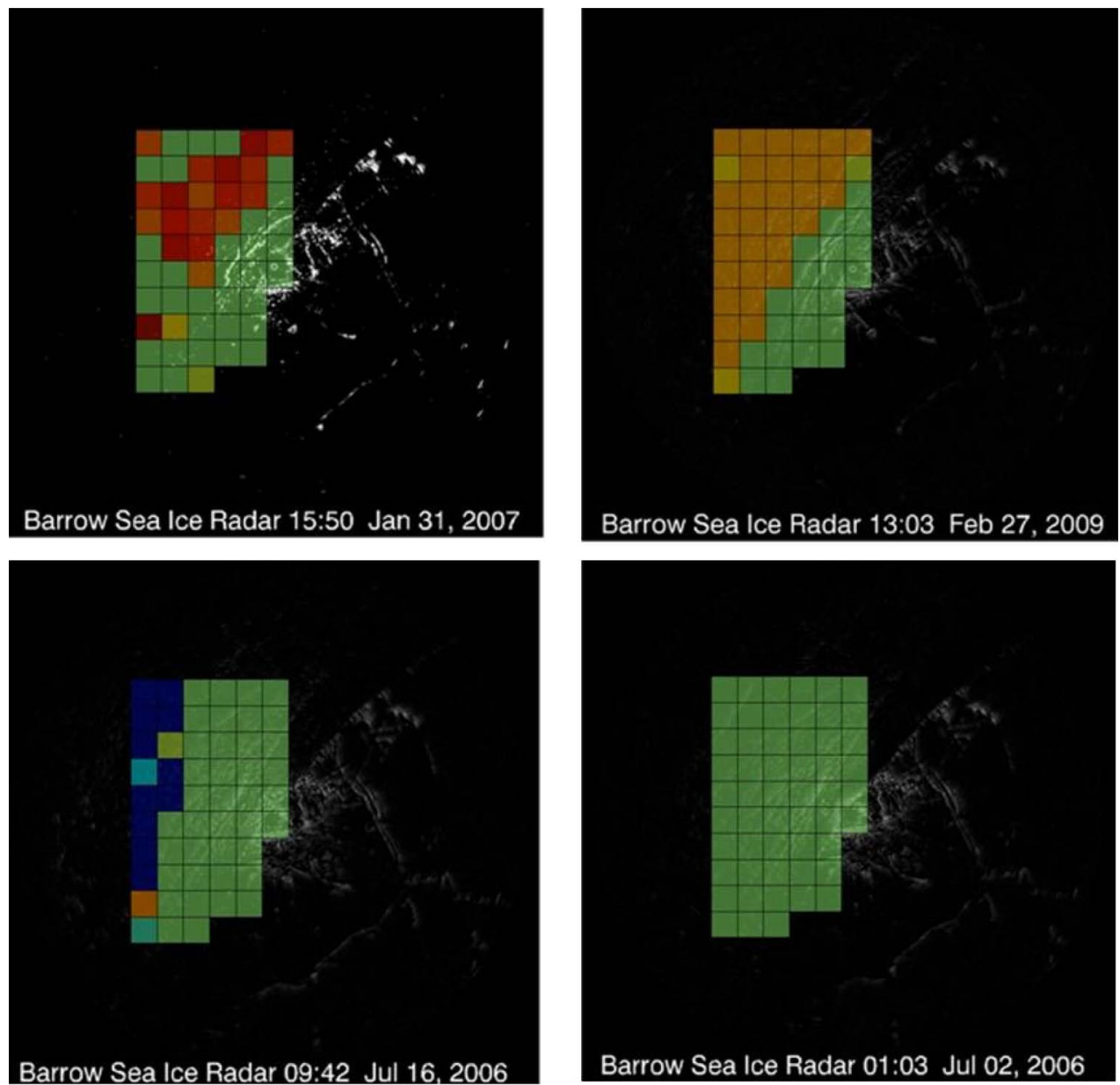

Fig. 10. Some examples of event detection. The top two images show a break-up event. The bottom two correspond to smooth flow and no flow. The colors are as per Fig. 6 .

regarded as the location of the boundary between stationary and moving ice. Since the satellite derived ice edges are not derived from the radar imagery, but from georeferenced SAR imagery, these images have been georeferenced to allow comparison of program and manually derived ice edges with satellite-derived edges. The radar images were referenced using pixel size-based methods, from which errors arise due to the non-uniform spatial sampling by the radar system. These errors are up to $1 \mathrm{~km}$ at a distance of $3.5 \mathrm{~km}$ from the center of the radar footprint, and increases with distance. Current work is under way to better geolocate the radar imagery using ground control points. Another source of error that may be present in all of these images is the quality of the radar imagery itself. The 2006-07 ice-season was the first during which the radar was in operation and the system had not yet been optimized for ice detection and noise reduction. 

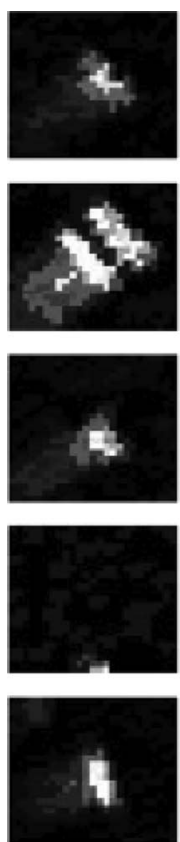

(a)
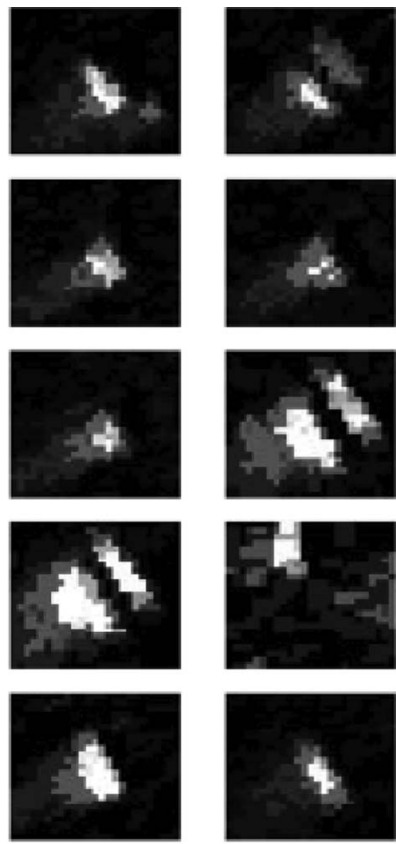
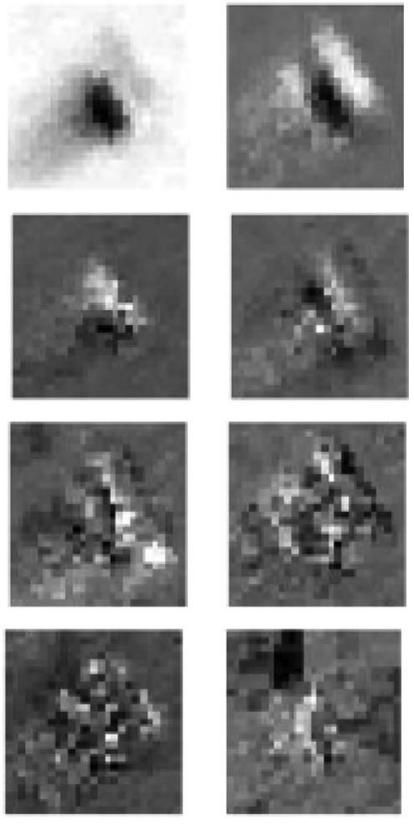

(b)

Fig. 11. (a) Samples of $20 \times 20$ pixel image patches centered around vessels. (b) The first eight principal components identified from the training data.

Fig. 12. Examples of detected vessels. The red box indicates the identified by our system as containing vessels. The blue box indicates the regions manually marked regions containing vessels. The right image has one false detection.

\section{Ice Hazard Event Detection}

The Viterbi algorithm building on the Hidden Markov Model as described above was able to correctly label most of the grid locations affected by anomalous or potentially hazardous ice events after training of the algorithm. Among seven examples of positive data (see Fig. 10), break-outs were not detected in two of them. In one of these cases, the break-out occurred along the margins of the radar footprint where few reflectors were detectable. The other case of failure to detect occurred when the break-out was associated with ice moving in a different direction from all other break-out events. Among the negative examples (Fig. 10, bottom), no false positives occurred. Due to the rarity of break-out events, more data needs to be ingested and used for model training to improve the performance of the algorithm. However, the sensitivity of the algorithm to correctly identify even the early stages of break-out events due to anomalous motion and oscillations in radar reflector signal strength [42] suggest that this approach is highly promising as a decision support and early-warning tool.

\section{Detection of Surface Vessels}

We used imagery from 15 days which contained 20 distinct vessels to train and test our vessel detection system. 48 frames were selected from those days at times when the vessels were visible. We divided the data into 24 frames of testing and 24 frames of training. A $20 \times 20$ pixel patch centered around a vessel was extracted from each frame of the training data. Principal component analysis (PCA) was performed to find the appearance model of the patches. Fig. 11 shows examples of 
sample patches and imagery. The first eight principal components captured about $90 \%$ of the variation of the sample set, indicating that the linear model was a suitable fit. Detection was performed on the testing frames by choosing candidate image patches using the blob filter and then projecting them onto the components determined using PCA. Two sample results are shown in Fig. 12. Of the 33 vessels contained in the 24 testing frames, our method was correctly able to detect 28 of them. It also detected 10 false vessel locations in those images. Lowering the threshold for detection resulted in a large number of false detections (nearly 50) without increasing true detection rate whereas increasing the threshold of detection did not cut down the number of false positives significantly. For use in an operational setup, our current system of vessel detection requires manual review. After obtaining radar imagery with dense temporal sampling, we will extend this method in our future work to include characteristics of vessel motion to increase the accuracy of detection.

\section{CONCLusion}

Ground-based radar systems offer several advantages in tracking surface processes or features compared to airborne or space based radars. These advantages include much higher sampling rates, stable configuration and short path delays in a system that is much more cost-effective. Detailed analysis has shown that current coverage of the region of interest by SAR data is on average roughly one scene per day which is not sufficient to most operational demands relevant in the context of emergency response [5]. However, SAR and other satellite data can provide a reference framework within which to interpret and analyze the data and information obtained from the radar system described here. Specifically, we have analyzed ground-based radar imagery of landfast and moving sea ice and developed algorithms to automatically extract tracks of reflectors and derive velocity fields for moving ice, perform landfast edge delineations, detect break-outs and other ice hazard events and identify surface vessels. We have used a combination of dense and sparse optical flow methods to estimate motion in the imagery. Using active contours, the landfast ice delineation is performed with little to no user intervention. The dynamic state of the ice is modeled by Hidden Markov Models and the state transitions are marked out as events associated with ice hazards. Surface vessel characteristics were obtained from principal component analysis of imagery containing known vessel reflectors and were then successfully applied for automated detection of vessels. A comparison of our methods with manual analysis of the imagery shows that the proposed methods perform well on real imagery. In this paper, we have proposed some automatic methods for analyzing ground based marine radar imagery. Using a combination of computer vision and machine learning techniques we demonstrated extraction of physical parameters and detection of anomalous events. For our future work, we propose to exploit better temporal sampling in radar imagery and extend our algorithms to improve the accuracy of event prediction and also derive more complex physical attributes of ice motion.

\section{REFERENCES}

[1] E. O. Lewis, B. W. Curry, and S. Haykin, Detection and Classification of Ice. New York: Wiley, 1987, p. 325.

[2] G. V. Shevchenko, A. B. Rabinovich, and R. E. Thomson, "Sea-ice drift on the northeastern shelf of Sakhalin island," J. Phys. Oceanogr., vol. 34, no. 11 , pp. 2470-2491, 2004.

[3] J. Briggs, "Target detection by marine radar," IEEE Aerosp. Electron. Syst. Mag., vol. 20, no. 6, pp. 39-40, Jun. 2005.

[4] L. W. Brigham, "The fast-changing maritime Arctic," Proc. U.S. Naval Inst., vol. 136, no. 5, pp. 55-59, May 2010.

[5] H. Eicken, J. Jones, C. Kambhamettu, F. Meyer, A. Mahoney, and M. L. Druckenmiller, "Environmental security in arctic ice-covered seas: From strategy to tactics of hazard identification and emergency response," Marine Technol. Soc. J., vol. 45, no. 3, pp. 37-48, May 2011.

[6] M. L. Druckenmiller, H. Eicken, M. A. Johnson, D. J. Pringle, and C. C. Willliams, "Towards an integrated coastal sea-ice observatory: System components and a case study at Barrow, Alaska," Cold Reg. Sci. Technol, vol. 56, no. 2/3, pp. 61-72, May 2009.

[7] C. Werner, T. Strozzi, A. Wiesmann, and U. Wegmuller, "A realaperture radar for ground-based differential interferometry," in Proc. IEEE IGARSS, Jul. 2008, vol. 3, pp. III-210-III-213.

[8] D. Leva, G. Nico, D. Tarchi, J. Fortuny Guasch, and A. Sieber, "Temporal analysis of a landslide by means of a ground-based sar interferometer," IEEE Trans. Geosci. Remote Sens., vol. 41, no. 4, pp. 745-752, Apr. 2003.

[9] G. Luzi, M. Pieraccini, D. Mecatti, L. Noferini, G. Guidi, F. Moia, and C. Atzeni, "Ground-based radar interferometry for landslides monitoring: Atmospheric and instrumental decorrelation sources on experimental data," IEEE Trans. Geosci. Remote Sens., vol. 42, no. 11, pp. 2454-2466, Nov. 2004

[10] R. Pilkington, A. Keinonen, V. Tambovsky, S. Ryabov, V. Pishchalnik, and A. Sheikin, "Ice monitoring program in support of Sakhalin energy's offshore oil production," in Proc. ICETECH, 2006, vol. 6, pp. 101-108.

[11] M. Wakatsuchi and K. I. Ohshima, "Observations of ice ocean eddy streets in the sea of Okhotsk off the Hokkaido coast using radar images," J. Phys. Oceanogr., vol. 20, no. 4, pp. 585-594, Apr. 1990.

[12] A. Mahoney, H. Eicken, and L. Shapiro, "How fast is landfast sea ice? A study of the attachment and detachment of nearshore ice at Barrow, Alaska," Cold Reg. Sci. Technol., vol. 47, no. 3, pp. 233-255, Mar. 2007.

[13] R. Kwok, A. Schweiger, D. A. Rothrock, S. Pang, and C. Kottmeier, "Sea ice motion from satellite passive microwave data assessed with ERS SAR and buoy data," J. Geophys. Res., vol. 103, no. C4, pp. 8191-8214, 1998.

[14] M. Thomas, C. Kambhamettu, and C. Geiger, "Motion tracking of discontinuous sea ice," IEEE Trans. Geosci. Remote Sens., vol. 49, no. 12, pp. 5064-5079, Dec. 2011

[15] C. Harris and M. Stephens, "A combined corner and edge detector," in Proc. 4th Alvey Vis. Conf., 1988, pp. 147-151.

[16] D. G. Lowe, "Distinctive image features from scale-invariant keypoints," Int. J. Comput. Vis., vol. 60, no. 2, pp. 91-110, Nov. 2004

[17] Q. Li, C. Kambhamettu, and J. Ye, "Integrating spatial and discriminant strength for feature selection and linear dimensionality reduction," in Proc. Conf. CVPRW, 2006, p. 21.

[18] N. Vasconcelos, Feature Selection by Maximum Marginal Diversity, S. Becker, S. Thrun, and K. Obermayer, Eds. Cambridge, MA: MIT Press, 2002, pp. 1351-1358.

[19] N. Vasconcelos and M. Vasconcelos, "Scalable discriminant feature selection for image retrieval and recognition," in Proc. CVPR, 2004, vol. 2, pp. II-770-II-775.

[20] M. Yeary, Y. Zhai, T.-Y. Yu, S. Nematifar, and A. Shapiro, "Spectral signature calculations and target tracking for remote sensing," IEEE Trans. Instrum. Meas., vol. 55, no. 4, pp. 1430-1442, Aug. 2006.

[21] M. Stakkeland, O. Hallingstad, and O. Overrein, "Tracking and fusion of surveillance radar images of extended targets," in Proc. 10th Int. Conf. Inf. Fusion, Jul. 2007, pp. 1-8.

[22] J. Spoelstra and E. C. Botha, "New rotation-invariant features for radar target recognition," Signal Process., vol. 55, no. 3, pp. 351-367, Dec. 1996.

[23] C. Weishi, N. Huansheng, and L. Jing, "Detecting and tracking of small moving target in avian radar images," in Proc. Int. Conf. IASP, Apr. 2010, pp. 473-476.

[24] A. Moqiseh and M. Nayebi, "3-D Hough transform for surveillance radar target detection," in Proc. IEEE Radar Conf., May 2008, pp. 1-5.

[25] K. Mikolajczyk and C. Schmid, "Scale \& affine invariant interest point detectors," Int. J. Comput. Vis., vol. 60, no. 1, pp. 63-86, Oct. 2004

[26] B. D. Lucas and T. Kanade, "An iterative image registration technique with an application to stereo vision (IJCAI)," in Proc. 7th IJCAI, 1981, pp. 674-679. 
[27] Y. Zheng, C. Kambhamettu, T. Bauer, and K. Steiner, "Accurate estimation of pulmonary nodule's growth rate in CT images with nonrigid registration and precise nodule detection and segmentation," in Proc. Comput. Vis. Pattern Recognit. Workshop, 2009, pp. 101-108.

[28] M. Bertalmio, G. Sapiro, and G. Randall, "Region tracking on surfaces deforming via level-sets methods," in Scale-Space Theories in Computer Vision, M. Nielsen, P. Johansen, O. Olsen, and J. Weickert, Eds. Berlin, Germany: Springer-Verlag, 1999, ser. Lecture Notes in Computer Science, pp. 330-338.

[29] M. Li, C. Kambhamettu, and M. Stone, "Automatic contour tracking in ultrasound images," Int. J. Clin. Linguist. Phonet., vol. 19, no. 6/7, pp. 545-554, Sep.-Nov. 2005.

[30] V. Caselles, R. Kimmel, and G. Sapiro, "Geodesic active contours," Int. J. Comput. Vis., vol. 22, no. 1, pp. 61-79, Feb./Mar. 1997.

[31] R. Malladi, J. Sethian, and B. Vemuri, "Shape modeling with front propagation: A level set approach," IEEE Trans. Pattern Anal. Mach. Intell., vol. 17, no. 2, pp. 158-175, Feb. 1995.

[32] X. Han, C. Xu, and J. L. Prince, "A topology preserving deformable model using level sets," in Proc. IEEE Comput. Soc. Conf. Compu. Vis. Pattern Recognit., 2001, vol. 2, pp. II-765-II-770.

[33] S. Osher and R. P. Fedkiw, "Level set methods: An overview and some recent results," J. Comput. Phys., vol. 169, no. 2, pp. 463-502, May 2001.

[34] J. E. Solem, H. Aanaes, and A. Heyden, "Variational surface interpolation from sparse point and normal data," IEEE Trans. Pattern Anal. Mach. Intell., vol. 29, no. 1, pp. 181-184, Jan. 2007.

[35] F. Porikli and X. Li, "Traffic congestion estimation using hmm models without vehicle tracking," in Proc. IEEE Intell. Veh. Symp., Jun. 2004, pp. 188-193.

[36] M. Li, C. Kambhamettu, and M. C. Stone, "Snake for band edge extraction and its applications," in Proc. IASTED Comput. Graph. Imag., 2003, pp. 261-266.

[37] Amini and A. Arsham, "Using dynamic programming for solving variational problems in vision: Applications involving deformable models for contours and surfaces," Ph.D. dissertation, University of Michigan, Ann Arbor, MI, 1990.

[38] J. Forney, "The viterbi algorithm," Proc. IEEE, vol. 61, no. 3, pp. 268278, Mar. 1973

[39] C. Fowler, W. Emery, and J. Maslanik, "Satellite-derived evolution of arctic sea ice age: October 1978 to March 2003," IEEE Geosci. Remote Sens. Lett., vol. 1, no. 2, pp. 71-74, Apr. 2004.

[40] J. Maslanik, unpublished.

[41] D. Norton and A. G. Gaylord, "Drift velocities of ice floes in Alaskas northern Chukchi Sea flaw zone: Determinants of success by spring subsistence whalers in 2000 and 2001," Arctic, vol. 57, no. 4, pp. 347-362, Dec. 2004

[42] A. Mahoney, H. Eicken, L. Shapiro, A. Graves, and P. Cotter, "Landfast sea ice extent and variability in the Alaskan Arctic derived from sar imagery," in Proc. IEEE Int. Geosci. Remote Sens. Symp., 2004, vol. 3, pp. 2146-2149.

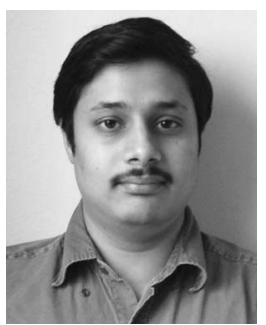

Rohith MV (S'11-M'12) received the B.E. degree in telecommunications from the PES Institute of Technology, Bangalore, India, in 2006. He is currently pursuing his Ph.D. at Video/Image Modeling and Synthesis Lab, University of Delaware, Newark.

He has worked in the area of 3-D reconstruction from stereo imagery and monocular video sequences, analysis of radar imagery and visualization of large scale numerical simulation results. His research interests include image processing, machine learning, and computer vision.

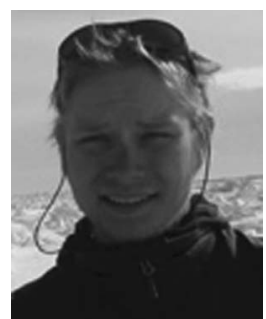

Joshua Jones is a graduate student in the Department of Geology and Geophysics at the University of Alaska, Fairbanks.

His research interests are focused on coastal sea ice dynamics, particularly concerning the formation of a stable landfast ice cover, evolution and deformation of landfast ice, and infrequent, but potentially hazardous, events such as break-outs.

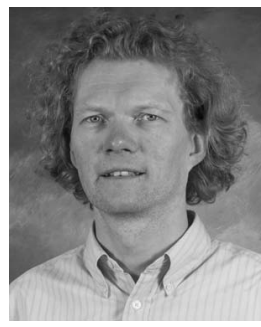

Hajo Eicken received his $\mathrm{Ph} . \mathrm{D}$. degree in natural Sciences from the University of Bremen, Germany, in 1990.

$\mathrm{He}$ is Professor of Geophysics at the University of Alaska, Fairbanks. His research interests include field studies of the growth, evolution, and properties of sea ice, as well as studies of ice use by people and its importance for ecosystems. Over the past decade, he has established a coastal sea-ice observatory in northern Alaska that provides an interface between geophysical and local knowledge of ice conditions and coastal hazards. Currently he heads an effort at the University of Alaska to enhance use of scientific data by a range of different stakeholders at the local and international level, building on work conducted during the International Polar Year.

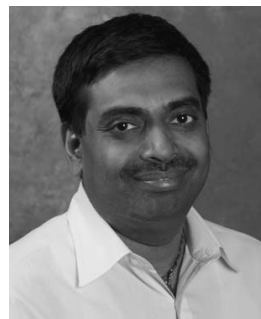

Chandra Kambhamettu is currently a Professor in the Department of Computer Science, University of Delaware, Newark, where he leads the Video/Image Modeling and Synthesis (VIMS) group. From 1994-1996, he was a Research Scientist at the NASA Goddard Space Flight Center (GSFC). His research interests include video modeling and image analysis for biomedical, remote sensing, and multimedia applications. He is best known for his work in motion analysis of deformable bodies, for which he received the NSF CAREER award in 2000. He has published over 200 peer-reviewed papers, supervised ten Ph.D. students and several Masters students in his areas of interest.

Dr. Kambhamettu received the Excellence in Research Award from NASA in 1995 while at GSFC. He has served as Area Chair, and has been technical committee member for leading computer vision and medical conferences. He has also served as Associate Editor for the journals Pattern Recognition and Pattern Recognition Letters and the IEEE TRANSACTIONS ON PATTERN ANALYSIS AND MACHINE INTELLIGENCE. 